Itinéraires Itinéraires

Littérature, textes, cultures

2012-3 | 2013

Lire les villes marocaines

\title{
Xavier Garnier et Jean-Philippe Warren (dir.), Les Écrivains francophones en exil à Paris : entre cosmopolitisme et marginalité
}

Paris, Karthala, 2012, 156 pages.

\section{Sada Soumaré et Marc Kober}

\section{CpenEdition}

\section{Journals}

Édition électronique

URL : http://journals.openedition.org/itineraires/1028

DOI : 10.4000/itineraires. 1028

ISSN : 2427-920X

\section{Éditeur}

Pléiade

Édition imprimée

Date de publication : 1 juillet 2013

Pagination : 176-181

ISBN : 978-2-343-01183-7

ISSN : $2100-1340$

\section{Référence électronique}

Sada Soumaré et Marc Kober, « Xavier Garnier et Jean-Philippe Warren (dir.), Les Écrivains

francophones en exil à Paris : entre cosmopolitisme et marginalité », Itinéraires [En ligne], 2012-3 | 2013,

mis en ligne le 01 décembre 2012, consulté le 22 septembre 2020. URL : http://

journals.openedition.org/itineraires/1028 ; DOI : https://doi.org/10.4000/itineraires.1028

Ce document a été généré automatiquement le 22 septembre 2020.

\section{cc)}

Itinéraires est mis à disposition selon les termes de la licence Creative Commons Attribution - Pas

d'Utilisation Commerciale - Pas de Modification 4.0 International. 


\section{Xavier Garnier et Jean-Philippe Warren (dir.), Les Écrivains francophones en exil à Paris : entre cosmopolitisme et marginalité}

Paris, Karthala, 2012, 156 pages.

Sada Soumaré et Marc Kober

\section{RÉFÉRENCE}

Xavier Garnier et Jean-Philippe Warren (dir.), Les Écrivains francophones en exil à Paris :

entre cosmopolitisme et marginalité, Paris, Karthala, 2012, 156 pages.

1 Publié sous la direction de Xavier Garnier et de Jean-Philippe Warren, l'ouvrage intitulé Écrivains francophones en exil à Paris : entre cosmopolitisme et marginalité aborde le rapport complexe entretenu avec cette ville par des auteurs venus du monde entier, ce qui permet de souligner des faits connus, notamment la dimension universelle de la langue française, ou le statut de «capitale de la république des lettres» de Paris durant une longue période. Certes, Paris fonctionne comme un modèle culturel et comme une instance de consécration. De ce fait, un premier niveau d'exil naît à distance, avant même d'y séjourner. Dans l'étude qu'il consacre à l'écrivain August Strindberg, Sylvain Briens revient sur une phrase de l'écrivain suédois : «Dans cinq ans je serai connu à Paris ! C'est la vengeance la plus cruelle que je puisse prendre sur les ânes et les bœufs. Elle est raffinée. » Sylvain Briens touche ici du doigt une des affirmations de Pascale Casanova, à savoir la capacité de la ville de Paris à consacrer mondialement un écrivain inconnu. Il explique le choix de l'écrivain: "Lorsque l'écrivain suédois August Strindberg s'installait à Paris en 1894, il fuyait, comme plusieurs de ses collègues, un milieu intellectuel suédois qui n'était pas encore prêt à comprendre la modernité de son œuvre» (p. 97). L'auteur de cette étude dépasse ce premier niveau, et avance une 
analyse fascinante de l'interaction entre l'espace urbain et un esprit en proie à une quadruple crise, psychologique, spirituelle, esthétique et scientifique, à travers la matière de récits intitulés Inferno et Légendes.

2 Au fil du temps, la ville devient fascinante. Régine Robin, qui apporte un vivant témoignage sur sa situation entre Paris et Montréal sous le titre «Rentre-t-on jamais chez soi ? ", évoque une "ville mythique ». C'est également cette puissance fascinatrice de la ville qui transparaît dans l'analyse de Claire Riffard. La plupart des auteurs évoqués ont découvert Paris grâce aux œuvres des auteurs français des siècles précédents qui ont su associer à la beauté de leurs textes sa splendeur. Parmi eux figurent Baudelaire, Hugo et Zola. Dans «Paris, capitale de l'intelligence!», texte consacré à J.-J. Rabearivelo, Claire Riffard se penche sur le rapport à la fois passionnel et livresque que le poète entretient avec la ville. Complètement séduit par le Paris découvert dans les œuvres de Baudelaire en particulier, cet auteur s'inspire en majeure partie de cette rencontre. À ce propos Claire Riffard déclare : «Parce qu'exilé à Paris, il l'a cependant été toute sa vie depuis qu'il a ouvert pour la première fois Les Fleurs $d u$ mal ou Le Spleen de Paris (qu'il écrit d'ailleurs sans italiques dans son journal, Les Calepins bleus, comme pour en souligner l'intime fréquentation: “N'ai-je pas moi-même, en effet, toujours, fait de ce spleen de Paris ma prière de tous les jours et de tous les instants ?")» (p.19). Rabearivelo ne connaîtra jamais l'expérience, difficile et enrichissante, du passage de la connaissance livresque à la connaissance vivante. Mais il comble ce désir inassouvi en imaginant Paris comme le "rêve d'une continuité géographique », mieux, comme le prolongement de sa vie d'intellectuel. L'étude de Claire Riffard présente un tout autre aspect de l'exil, à savoir un exil qui vise uniquement la quête de l'intelligence. Si Rabearivelo en reste à cette fascination lointaine, et sera incapable de rencontrer réellement la ville de Paris, de nombreux intellectuels francophones étrangers vont au-delà des textes et s'y rendent physiquement. Dès lors, les descriptions romanesques se heurtent à la réalité. Les écrivains en exil passent du statut de lecteur à celui d'auteur et apportent un précieux témoignage sur le sens de cette expérience. Ils décrivent une expérience personnelle, celle de la déambulation et de l'errance. Les études qui composent ce volume insistent sur un aspect moins bien connu que la valeur globale de Paris dans la vie littéraire et artistique du siècle passé : l'impact que la rencontre avec cette ville a pu avoir sur leur vie privée et sur leurs œuvres. L'intérêt de cet ensemble d'études consacrées spécifiquement à tel ou tel écrivain est de montrer plus en détail comment l'exil à Paris (et pas ailleurs) remet le monde en perspective, pour reprendre l'expression des auteurs à l'origine de ce projet.

3 En effet, dans un second temps, le « mythe de Paris » qui génère la souffrance de ne pas y être, se lézarde, et c'est alors le sentiment d'exil par rapport au lieu d'origine qui se développe. Cet « exil au second degré » est l'objet principal du livre. Les textes réunis ici permettent d'illustrer ce que l'on savait déjà : l'exil est un moteur de création. Le nombre et la qualité des œuvres produites par certains auteurs durant l'exil parisien sont éloquents. Mais l'intention de cet ouvrage est de mettre à jour « un type d'exil particulier, attaché à la ville de Paris » (p.9). En particulier, les auteurs sont moins exilés en raison d'un dépaysement lié à la ville, qu'en raison du travail mental qu'elle les oblige à effectuer. Ils développent en effet une " hyperconscience du lieu natal », ou des autres capitales dont ils sont originaires au contact de Paris. 
C'est le cas des intellectuels québécois qui reviennent au Québec après un exil parisien. Cette situation, désignée sous le nom de "retour d'Europe», est étudiée par JeanPhilippe Warren et Michel Lacroix. Le point commun entre ces intellectuels est leur fascination pour la ville où ils ont séjourné. La grandeur de Paris les transforme au point d'avoir du mal à vivre dans leur pays d'origine. Les auteurs de cette étude reviennent sur une déclaration d'André Laurendou. « Règle générale, écrivait-il, ils (les intellectuels québécois de retour d'Europe) me semblaient insupportables, jamais satisfaits de ce qu'ils trouvaient ici, injustes et cassants, toujours à la recherche de comparaisons odieuses; ou bien inadaptés, ayant perdu le sens du possible; ou bien bâclant leur tâche et se taisant, avec une préoccupation: retourner là-bas, en rêve sinon en réalité » (p. 50). Ce n'est qu'après un séjour dans la capitale française qu'André Laurendou finit par comprendre la particularité de l'expérience parisienne : «Il [le visiteur canadien] connaît là-bas, ou du moins il frôle, il entrevoit la grande aventure intellectuelle. C'est un alcool qui monte à la tête. C'est trop fort pour lui. Ses défenses craquent de toutes parts» (p. 51). Pour Jean-Philippe Warren et Michel Lacroix, cette confrontation avec Paris change la donne, car " [i]ci le scénario auctorial de l'aller et retour Canada-France change de sens et d'extension: ce n'est plus le départ qui est présenté comme un exil, mais le retour» (p. 58). Parallèlement, les intellectuels se voient pris dans un éternel exil, ayant échoué à se frayer un chemin dans la grande métropole, ils envisagent le retour au pays natal comme un nouvel exil. Dans le même Québec, le regard que Régine Robin pose sur Montréal est influencé par l'image de Paris. Dans cette première confrontation, Montréal comparé à Paris ressemble à un village « La Parisienne que j'étais, en dehors du centre-ville, tout avait l'air de villages, d'une ville à la campagne, selon la jolie formule d'Alphonse Allais » (p. 150). Tout en relatant son enfance à Paris, elle affirme : «Aucun pays au monde ne pouvait rivaliser avec l'universalisme français» (p.144). Cette difficulté à vivre sans se référer constamment à la ville de Paris est oppressante. Paris devient une seconde demeure. " Je ne suis jamais tout à fait partie » reconnait Régine Robin.

5 C'est cette difficulté que Marina Muresanu Ionescu rappelle dans son analyse de l'écrivain Eugène Ionesco, "Le Paris de Ionesco: enfer ou paradis ou "Parisien de Roumanie" ". Elle décrit un homme tiraillé. Issu d'une mère française et d'un père roumain, Ionesco s'attache particulièrement à la ville de Paris grâce à sa mère, sinon pour sa mère. En faisant mention d'un témoignage de l'auteur, elle révèle le rapport symbolique que l'écrivain franco-roumain entretient avec la ville de Paris. "Tout ce que j'ai fait, c'est en quelque sorte contre lui que je l'ai fait. J'ai publié des pamphlets contre sa patrie (le mot patrie n'est pas supportable puisqu'il signifie le pays du père); mon pays était pour moi la France, tout simplement parce que j'y avais vécu avec ma mère, dans mon enfance, pendant les premières années de l'école et parce que mon pays ne pouvait être que celui dans lequel vivait ma mère " (p. 85). Renvoyant tour à tour aux exils de Ionesco, Marina Muresanu Ionescu fait ressortir les nombreuses impressions que Ionesco a pu ressentir, tout au long de son va-et-vient entre le pays du père tant détesté et celui de la mère tant chérie. Cet attachement à Paris, qui devient à la longue un amour tyrannique, va pousser l'écrivain à déclarer son souhait de voir la ville rester intacte, sans le moindre changement de façade, parce qu'elle symbolise le siège de son enfance, de son passé diplomatique mais encore plus important de sa vie d'artiste. Mais remarquant l'effet inverse, il ne cache pas son impuissance : «Paris se dégrade de plus en plus. Ouille, ouille, ouille ! Je suis pris au piège, j'ai tant voulu y vivre et, hélas, "écrire" » (p. 95). M. Ionescu commente ainsi ce cri de désespoir : «En effet, 
pendant toute sa vie, Ionesco aura été le prisonnier d'un cercle infernal, où de fulgurantes éclaircies paradisiaques alternent avec le désespoir le plus sombre, mais dont Paris, "Reine-e-e du monde", demeure toujours le centre. "

6 Paris est, au $\mathrm{xx}^{\mathrm{e}}$ siècle, un lieu de passage important pour les auteurs orientaux. En analysant les œuvres de deux d'entre eux, Tewfik al-Hakîm et Elian J. Finbert, Marc Kober souligne l'un des motifs de cet exil. "Le séjour en France est une manière d'activer son génie personnel» (p.35). Tout comme, J.-J. Rabearivelo évoque ces amours, Paris et Tananarive, les écrivains orientaux quant à eux, reconnaissent cette expérience parisienne comme faisant partie intégrante de leur personnalité. Le narrateur d'Elian J. Finbert accepte cette scission unitaire : «Comme le canal qui sépare deux continents, je suis moi aussi départagé. Il n'est plus possible de croire que je suis seulement oriental » (p. 37). Selon M. Kober, «[s]'il ne décrit pas sa vie d'exilé parisien, il en restitue indirectement le déchirement et son partage entre deux "patries". La première image convoquée au moment du départ de cet "homme de l'Orient" est celle du canal à Port Saïd». Quant à Tewfik al-Hakîm, il le décrit comme "[...] un immense lecteur, et un amant contrarié par les aspirations de femmes incompatibles avec sa quête esthétique ». Tewfik al-Hakîm répond aux critères mêmes de l'auteur marginal, celui qui n'arrive pas à s'ouvrir un passage dans le milieu parisien. Mais le séjour parisien a certainement été déterminant, même a contrario, dans la réussite de sa carrière d'homme de lettres en Égypte, puisqu'il est devenu extrêmement célèbre grâce à son œuvre théâtrale, et grâce à certains récits ancrés dans la réalité égyptienne. La tentation cosmopolite et parisienne a fait long feu. S'il peine à construire une vie sociale heureuse, il profite néanmoins de la richesse culturelle de la ville. C'est un livre dans lequel le narrateur, à l'image de Tewfik al-Hakîm, fréquente les endroits culturels parisiens mais à un détail près. Le narrateur de L'Oiseau d'Orient sent sa petitesse face à la grandeur des institutions culturelles prestigieuses, ou plus ordinaires, mais avant tout parisiennes. Il développe un fort complexe d'infériorité. Cette œuvre est l'occasion d'une nouvelle approche de la ville: "Ce roman est original dans la mesure où le monde des usines et des banlieues est très rarement représenté à cette époque, tandis que se maintient une image stéréotypée de la capitale. Bien au contraire, c'est la fraternité virile avec un exilé russe, à mi-chemin entre l'Orient et l'Occident, qui domine la seconde moitié du roman » (p. 42).

7 La rencontre des écrivains africains avec l'ex-métropole, inscrite dans une perspective postcoloniale n'échappe pas à cette phase de fascination de la ville. Xavier Garnier suit pour son analyse la matière de trois œuvres principales de la littérature francophone d'Afrique noire : L'Aventure ambiguë, Un Nègre à Paris, et Mirages de Paris. Il revient sur les différentes étapes que comporte la confrontation avec Paris, de la phase d'enthousiasme à la phase d'individualisation et enfin la phase de fraternisation avec les autres peuples. Selon Xavier Garnier, cette dernière phase intervient lorsque l'intellectuel francophone africain constate sa grande différence avec le peuple français. Le plus souvent il se retrouve dans la marginalité car incapable de sortir de l'ombre pour entrer au grand jour de la «Ville-lumière ». Pourtant, cette émergence à la lumière est le propre de l'aventure des Noirs, des souterrains du métropolitain de Paris à l'underground new-yorkais, par la médiation du jazz en particulier.

8 Dans cette aventure parisienne, la langue française est également source de tension. Cet ouvrage apporte une nuance intéressante, en distinguant le francophone étranger $\mathrm{du}$ francophone tout court. Au fond, le francophone étranger se retrouve dans la 
situation d'un provincial fraîchement monté dans la capitale. Il est dérouté par l'espace social et physique rencontré, mais surtout, il conserve un accent, et parfois une langue autre. Les expatriés du dedans de la francophonie ressemblent à ces expatriés du territoire national centralisé. L'idée si répandue de l'universalité de la langue française se heurte à des situations beaucoup plus retorses dans la réalité, entre francophones de langue maternelle et auteurs qui choisissent le français comme langue d'écriture. L'exil des écrivains francophones étrangers à Paris est perçu comme un « exil intérieur » car ce qui les sépare semble être la distance géographique et non la langue. Mais en réalité, l'apparente " patrie linguistique » de la langue française n'est qu'un leurre qui creuse le sentiment d'exil. C'est une patrie cosmopolite, une terre d'adoption, mais qui n'exclut pas les ruptures.

9 Dominique Combe décrit, avec "L'exil parisien de Charles-Ferdinand Ramuz », un homme déchiré et en conflit avec la langue française. Si Ramuz a su se faire une place à Paris, il n'en reste pas moins qu'il éprouve une absence de spontanéité dans le parler des francophones étrangers. Il conteste l'hégémonie de la langue parlée à Paris. Dans une partie intitulée "L'exil linguistique ", il revient sur la position qu'adopte l'écrivain et sur sa situation d'écrivain francophone étranger: "L'exil tient certes d'abord au sentiment aigu d'être dans "l'entre-deux" (Daniel Sibony): "Étranger" par son passeport, ce très célèbre écrivain de suisse romande se définit comme "français par la langue, et par la langue seulement" »(p.72). Là encore, la situation de l'écrivain francophone, dans sa relation avec Paris, oscille entre identification, rejet ou sublimation.

10 C'est également le rapport à la langue que Mireille Calle-Gruber met en relief dans son analyse de l'écriture d'Assia Djebar, qui prend pour thème principal l'exil linguistique, celui qui s'effectue à l'intérieur de la langue française: «J'écris dans l'ombre de ma mère revenue de ses voyages de temps de guerre, moi, poursuivant le mien dans cette paix obscure faite de sourde guerre intérieure, de divisions internes, de désordres et de houle de ma terre natale » (p. 131). En s'appuyant sur l'ouvrage Vaste est la prison d'Assia Djebar, Mireille Calle-Gruber démonte le processus par lequel l'auteure suggère la nature culturelle de la ville de Paris. La vie intellectuelle se manifeste jusque dans les moindres aspects de la capitale. "Paris est lieu d'éducation ("la pension d'étudiants"). L'espace des chambres a tout de même la noblesse de la "librairie" et des "multiples bibliothèques", ce qui donne à "l'obscurité le prestige du secret à lire et découvrir" " (p. 131).

11 Ce volume de "traversées de l'exil» au prisme d'une ville unique, Paris au xxe siècle, met bien en valeur à la fois le caractère ambivalent de la lumière de cette ville : on s'y brûle, mais c'est aussi à son contact que s'enclenche la création littéraire. Elle renforce l'imaginaire d'une langue universelle, et dans la pratique, rejette des écrivains pas assez exotiques, qui eux, ont souvent rompu tous les ponts avec un terroir linguistique. L'espace fascinant de la grande métropole coloniale, d'un cœur du monde, qui s'est rêvé (et qui a été perçu) comme le lieu de convergence de toutes les cultures, exacerbe les blessures et les dissonances. Entre le Paris rêvé à distance, médiatisé par tant de lectures préalables, et le Paris arpenté comme un territoire inattendu, se creuse une prise de conscience pleine de désillusion, mais aussi de lucidité. Tout est question d'échelle relative et de positionnement entre le local et l'universel. Dès lors, Paris, sa langue et sa culture deviennent du dernier provincial. Et pourtant, Paris ne cesse 
d'attirer à lui les amateurs de sa matérialité urbaine, en quête d'une révélation et d'une initiation qui passent par la perte de soi et par l'exil. 